\title{
Analysis of small nucleolar RNAs (snoRNAs) associated co-expression and construction of ceRNA regulatory networks in esophageal carcinoma
}

\author{
Haigen Jiang \\ Huzhou Central Hospital \\ Jing Zhuang \\ Huzhou University \\ Xi Yang \\ Huzhou Central Hospital

\section{Wei Wu} \\ Huzhou Central Hospital
}

\section{Siqi Dai}

Zhejiang University School of Medicine Second Affiliated Hospital

\section{Quang Qi}

Huzhou Central Hospital

Shuwen Han ( $\square$ shuwenhan985@163.com )

Huzhou Central Hospital https://orcid.org/0000-0001-6180-9565

\section{Research}

Keywords: snoRNAs, Esophageal carcinoma, SNHGs, Co-expression analysis, Survival analysis, ceRNA network

Posted Date: March 28th, 2020

DOI: https://doi.org/10.21203/rs.3.rs-19180/v1

License: (c) (i) This work is licensed under a Creative Commons Attribution 4.0 International License. Read Full License 


\section{Abstract}

Purpose: This study aimed to investigate the role of small nucleolar RNAs (snoRNAs) and their host genes (SNHGs) in tumorigenesis and progression of esophageal carcinoma (EC).

Methods: RNA-seq data and clinical information for EC patients were obtained from The Cancer Genome Atlas database. Differentially expressed snoRNAs (DE-snoRNAs) and SNHGs (DE-SNHGs) between EC samples and normal controls were screened with the edgeR package, followed by the trend analysis with STEM. Survival analysis was performed using the Kaplan-Meier method with the log-rank test. Screening of co-expressed genes and functional enrichment analyses were performed with Pearson's correlation analysis and enrichment analysis, respectively. Lastly, the co-expression and ceRNA networks were constructed.

Results: A total of 19 DE-snoRNAs and nine DE-SNHGs were screened between EC samples and normal controls. Moreover, two snoRNA clusters related to the clinic stage were analyzed through trend analysis. Survival analysis results demonstrated that SNORA70D was significantly associated with the overall survival of EC patients $(P=0.034)$. In addition, a co-regulation network that included six snoRNAs and 29 mRNAs was constructed. A ceRNA network comprised of five SNHGs, 11 mRNAs, and 38 miRNAs was constructed based on the top 50 relationship pairs. KEGG pathway enrichment analysis revealed that SNORA14B, SNORA47, SNORA71C, SNORD12B, and SNORD14E in the co-expression network were mainly enriched in the NOD-like receptor signaling pathway, and neuroactive ligand-receptor interaction pathway. SNHG23, SNHG3, SNHG17, SNHG4, and SNHG8 in the ceRNA regulation network were mainly enriched in calcium signaling pathway, cytokine-cytokine receptor interaction, and the extracellular matrix-receptor interaction pathway.

Conclusion: The data revealed a series of snoRNAs, SNHGs, and pathways involved in EC carcinogenesis, which will provide a basis for understanding the underlying molecular mechanism of EC development.

\section{Highlights}

1. A series of DE-snoRNAs and DE-SNHGs were screened.

2. Candidate pathways associated with snoRNAs or SNHGs were enriched.

3. Co-expression and ceRNA networks associated with EC were conducted.

\section{Introduction}

Esophageal carcinoma (EC), one of the most aggressive cancers, has been the sixth leading cause of cancer deaths worldwide, representing the $3.2 \%$ of all cancers [1,2]. Traditionally, EC is subdivided into two histologic types, including esophageal squamous cell carcinoma (ESCC) and esophageal adenocarcinoma (EAC), of which $88 \%$ of the cases are ESCC [3]. Accumulating evidence shows that the risk factors of EC include smoking, alcoholic beverages, Barrett's esophagus, gastric reflux and obesity 
$[4,5]$. Till now, despite the improvements of multidisciplinary treatments, such as surgery, chemotherapy, and radiotherapy, the prognosis of EC patients still remains poor, with the 5-year survival rate remains only approximately $20 \%$ [6]. To our knowledge, EC is a multistep process involving a series of genetic or epigenetic alterations; hence, to investigate the molecular and pathogenic mechanisms underlying the carcinogenesis of EC is still necessary.

Small nucleolar RNAs (snoRNAs), comprising of 60-300 nucleotides in length, are a conserved class of non-coding RNAs that contribute to ribosome biogenesis and RNA splicing by modifying ribosomal RNA and spliceosomal RNAs. The role of snoRNA in tumor development was first reported in 2002 [7]. In the ensuing decade, an increasing number of studies have indicated the involvement of snoRNAs in different tumors, including lymphoma, breast cancer, melanoma, and myeloma [8-12]. It has been reported that snoRNAs are regulated in a was complex manner by host genes, copy number variation, and DNA methylation [13]. As the host genes for snoRNAs, small nucleolar RNA host genes (SNHGs) include coding genes and non-coding genes. Long non-coding small nucleolar host genes are one of the classes of SNHGs. Increasing Inc-SNHG members continue to be identified in cancers. For instance, it has been reported that SNHGs are involved in the development of various diseases, including cancer progression, cell apoptosis, and survival [14]. SNHG8 may play oncogenic roles in the malignancy of ESCC, and offer potential biomarkers for the diagnosis and prognosis of ESCC [15]. LncRNA SNHG1 may be a potential predictor of prognosis in ESCC patients and a novel target for ESCC treatment [16].

With the development of microarray profiles and RNA-sequencing, a series of public gene expression analyses were published, including The Cancer Genome Atlas (TCGA; https://portal.gdc.cancer.gov/) and Gene Expression Omnibus (GEO; https://www.ncbi.nlm.nih.gov/gds) datasets. In particular, some snoRNAs and SNHGs play important roles in biological processes of disease. However, few studies have addressed the presence of these snoRNAs or SNHGs in EC. Hence, in the present study, we conducted a bioinformatics analyses to screen the candidate snoRNAs, SNHGs, and pathways involved in EC development as shown in Supplementary Figure 1.

\section{Materials And Methods}

\section{Data collection and preprocessing}

Public RNA-sequencing data and survival data of patients with EC were downloaded from the UCSC Xena [17] ( https://xenabrowser.net/). The data are open to the public under certain guidelines. All expression profiles data were classified into two groups: normal and tumor. Clinical information, including age at diagnosis, gender, site, type and body mass index was summarized in Table 1. After the extraction of snoRNAs and SNHGs data, the initial log (counts+1) value was converted to the counts.

\section{Differential snoRNAs and SNHGs expression analysis}

Based on the read count, differentially expressed snoRNAs (DE-snoRNA) and SNHGs (DE-SNHGs) were analyzed using the TMM (trimmed mean of $\mathrm{M}$ values) normalization method of edgeR software package 
$[18,19]$, followed by difference analysis using quasi-likelihood (QL) F-test method. The cutoff criteria of DE-snoRNA was $P<0.05$ and $|\log F C|>1$ (foldchange $>1.2$ ), and that for $D E-S N H G$ s screening was $P<0.05$ and $|\log \mathrm{FC}|>0.585$ (foldchange $>1.5$ ). Hierarchical clustering analysis was performed for the DE-snoRNA and DE-SNHGs using the R package.

\section{Trend analysis}

According to the diagnosis classification of tumor stage, EC was divided into four stages, including stage i, stage ii, stage iii, and stage iv. The trend analysis was performed with STEM[20] (version 1.3.11, http://www.cs.cmu.edu/ jernst/stem/).

\section{Screeningof the host genes of the snoRNA and functional annotation}

The host genes of the DE-snoRNAs and snoRNAs related to clinical stage of EC were extracted by using the snoopy tool (http://snoopy.med.miyazaki-u.ac.jp/)[21]. For functional annotation of those host genes of snoRNA, GO and KEGG pathway annotations were conducted with the cluster profiler package [22]. Significant pathways with a threshold of $P<0.05$ were selected.

\section{Survival analysis between snoRNA and methylation}

According to the TCGA datasets, Kaplan-Meier analysis was used to screen the genes showing clinical relevance in tumor patients. Briefly, the samples were divided into two groups according to the median expression of each gene: high expression and low expression groups. The log-rank test was used to analyze the differences between groups, and the significantly prognostic value was selected with a threshold of $\mathrm{P}<0.05$. Statistical analyses were performed using R package ("survival").

\section{Correlation analysis between snoRNA and methylation}

Methylation has been regarded as a key factor in the abnormal expression of snoRNA in cancers. The correlation analysis of the methylation level and DE-snoRNA was analyzed by the SNORic data portal ( http://bioinfo.life.hust.edu.cn/SNORic/) [22].

\section{Construction of snoRNA-mRNA co-expression network and ceRNA regulation network}

Based on the expression values of snoRNA or trend-related snoRNA, as well as the expression values of differentially expressed mRNAs corresponding to diseases, a correlation analysis was conducted to obtain the mRNAs corresponding to snoRNAs, with the threshold of $P<0.05$ and $|r|>0.4$. Furthermore, the enrichment analysis of the snoRNA-mRNA co-expression pairs were further carried out using the cluster profiler package [22].

To construct the ceRNA regulation network, Pearson correlation analysis was performed to determine the correlation of the DE-SNHG and DEGs (threshold: $P<0.05$ ). The top 5 members of the IncRNA-mRNA relationships were selected for further study. Subsequently, the targeted miRNAs of IncRNAs and mRNAs were predicted based on the MiRanda software [5]. Next, the ceRNA network was constructed based on 
the IncRNA-miRNA and IncRNA-mRNA relationship pairs with the threshold of -sc 140 and -en -20.

Similarly, the enrichment analysis of the genes in the network was done using the cluster profiler package [22].

\section{Statistical analyses}

All statistical analyses were performed using GraphPad Prism 7.0 (GraphPad Inc., La Jolla, CA), and all data are expressed as the mean \pm standard deviation (SD). Statistical comparisons between groups of normalized data were performed using the Student's t-test. $\mathrm{P}<0.05$ was considered to be statistically significant difference.

\section{Results}

\section{Screening of DE-snoRNA and DE-SNHGs}

Based on the fold change of $\mathrm{P}<0.05$ and $|\log \mathrm{FC}|>1$, a total of 19 snoRNAs were revealed to be differentially expressed between EC and normal controls. Among these DE-snoRNAs, 16 were upregulated and 3 were down-regulated in EC compared with normal samples (Supplementary Table 1). In addition, compared with those in the normal, there were 9 DE-SNHGs, including 4 up- and 56 downregulated $\mathrm{SNHGs}$ in the EC samples, with the threshold of $\mathrm{P}<0.05$ and $|\log \mathrm{FC}|>0.585$. The heat map of hierarchical clustering showed that the DE-snoRNAs or DE-SNHGs could be significantly distinguished between two groups (Figure 1)

\section{Screening of snoRNAs and SNHGs related to clinical stage}

As shown in Figure 2A, the expressed trends of snoRNAs related to clinical stage of EC were further analyzed. Two snoRNA clusters, including cluster 6 and cluster 7 were screened. A total of 22 and 169 snoRNAs were found in cluster 6 and cluster 7, respectively (Supplementary File 1). The expressed trends of the snoRNAs in two clusters are illustrated in Figure 2B and C. However, no SNHG cluster was significantly related to the clinical stage of EC.

\section{Functional annotation}

To analyze the functions of the DE-snoRNAs and snoRNAs related to clinical stage of EC, the host genes were screened (Table 2). As shown in Figure 3A, the host genes, including SNORA14A, SNORD11B, SNORD15A, SNORD53, and SNORD72, were mainly enriched in the GO BP terms related to regulation of steroid hormone biosynthetic process, positive regulation of cholesterol metabolic process, and carnitine metabolic process. Furthermore, KEGG pathway enrichment analysis revealed that SNORD11B, SNORD15A, SNORD53 and SNORD72 were involved in pathways that included Ribosome biogenesis in eukaryotes, Ribosome and n Pathogenic Escherichia coli infection (Figure 3B). The host genes of clinical stage-related snoRNAs (SCARNA10, SCARNA12, SNORA1, and SNORA11) were mainly enriched in pathways that included mitotic chromosome condensation, chromosome condensation, and mitotic sister chromatid segregation (Figure $3 \mathrm{C}$ ). 
To predict the relationship between snoRNAs and methylation, correlation analysis was performed based on the methylation levels of the EC samples from the TCGA. The results showed 7 DE-sonRNAs and 5 snoRNAs related to clinical stage were found significantly correlated with the methylation levels (all, $\mathrm{P}<0.05$; Figure 4 and Supplementary table 2), respectively.

Survival analysis of DE-snoRNAs and snoRNAs related to clinical stage of EC

In order to screen the snoRNAs, which may show clinical relevance with tumor patients, we performed the survival analysis on the DE-snoRNAs and snoRNAs related to clinical stage using TCGA datasets.

Kaplan-Meier curve showed that patients with high expression of SNORA70D had shorter overall survival compared to those with low expression ( $P=0.034$; Figure 5), suggesting SNORA70D is associated with the prognosis of EC patients.

Construction of snoRNA -mRNA co-expression network

A total of 38 and 3 DEGs were respectively found to be associated with DE-sonRNAs and clinical staging related snoRNAs based on the Pearson correlation coefficient analysis. The co-regulation network of snoRNA-mRNA was shown in Figure 6A, which included 6 snoRNAs and 29 mRNAs. Among these snoRNAs, SNORD12B was found to have the highest degree (degree=12). Furthermore, KEGG pathway enrichment analysis of snoRNA -mRNA relation pairs (top 5) revealed that SNORA14B, SNORA47, SNORA71C, SNORD12B, and SNORD14E mainly enriched in transcriptional misregulation in cancer, NOD-like receptor signaling pathway, neuroactive ligand-receptor interaction (Figure 6B).

Construction of the ceRNA regulation networks

According to the miranda software, IncRNA-miRNA and mRNA-miRNA relationship pairs were obtained, respectively (Supplementary file 2). Next, the ceRNA network was constructed using the top 50 relationship pairs. As illustrated in Figure 7A, the ceRNA network was composed of 5 SNHG nodes, 11 mRNA nodes, and 38 miRNA nodes. Subsequently, KEGG pathway enrichment analysis of SNHGs (top 5: SNHG23, SNHG3, SNHG17, SNHG4 and SNHG8) revealed a series of pathways, such as calcium signaling pathway, IL-17 signaling pathway, cytokine-cytokine receptor interaction and ECM-receptor interaction (Figure 7B).

\section{Discussion}

With the advances in high throughput sequencing, an increasing number of snoRNAs have been screened and are emerging as important RNAs, thereby attracting the attention of researchers. Studies have shown that some snoRNAs play important roles in biological processes, and dysfunction of snoRNAs may lead to oncogenesis [16]. It has also been reported that snoRNAs could serve as biomarkers in several diseases, including cancers [23]. Concerning the host gene for snoRNAs, SNHG genes may have diverse regulatory effects on cellular processes in some cancers $[24,25]$. In the present study, a total of $19 \mathrm{DE}-$ 
snoRNAs and 9 DE-SNHGs were screened between EC samples and normal controls, among which SNORA14B, SNORA47, SNORA71C, SNORD12B, and SNORD14E were selected as the key members due to their topological characteristics in the snoRNA-mRNA co-expression network; Similarly, SNHG23, SNHG3, SNHG17, SNHG4, and SNHG8 was selected as the key members in the ceRNA regulation network. SNORA47 is involved in lung cancer tumorigenesis [26] and promotes tumorigenesis by regulating EMT markers in hepatocellular carcinoma [27]. SNORA71C is overexpressed in breast cancer brain metastases [28]. Furthermore, SNHG3 facilitates cell proliferation and migration in oral squamous cell carcinoma via targeting nuclear transcription factor $Y$ subunit gamma [29]. It has been also been reported to that SNHG3-related ceRNAs can be potential research targets to explore the molecular mechanisms of HCC [30]. Moreover, SNHG17 is involved in gastric cancer, non-small-cell lung cancer, or melanoma through regulation of pathways, such as the phosphoinositide 3-kinase (PI3K)-AKT pathway [31-33]. However, until, little has been published of these snoRNAs or SNHGs in EC.

Oncogenesis is a complex process that is involved in the interaction of various genes and signaling pathways. Hence, the analysis of candidate snoRNAs or SNHGs and pathways related to EC could provide the cognitive basis for disease development. The present data reveal the key snoRNAs or SNHGs in co-expression and ceRNA networks mainly enriched in pathways, such as the NOD-like receptor signaling pathway and neuroactive ligand-receptor interaction, as well as calcium signaling pathway, cytokine-cytokine receptor interaction, and ECM-receptor interaction. Actually, cytokine-cytokine receptor interaction, NOD-like receptor signaling pathway, and ECM-receptor interaction are chief contributors to EC progression [34]. Zeng et al. [35] reported that the calcium signaling pathway and the neuroactive ligand-receptor interaction are the most relevant pathways in EC diagnosis using miRNA-seq and RNA-seq data. In accordance with previous studies, our data suggest the involvement of snoRNAs or SNHGs in the progression of EC by the aforementioned pathways.

Recent studies have suggested that snoRNAs can be a novel prognostic biomarkers and therapeutic targets for cancers. For instance, Zhu et al. [36] reported that SNORD89 deleteriously affects the prognosis of ovarian cancer patients by regulating the Notch1-c-Myc pathway. Another study described that the inhibition of SNORA7B expression impaired cell growth, proliferation, migration, and invasion by inducing apoptosis [37]. In this study, based on data from TCGA, patients with high expression of SNORA70D displayed shorter overall survival compared to those with low expression. However, the role of SNORA70D has not been reported in any disease, and it will be necessary to further validate the clinical prognostic value of SNORA70D EC. In addition to their host genes, snoRNAs can participate in the regulation of methylation. In EC, several genes with aberrant DNA methylation have been selected as potential disease biomarkers [38, 39] But until now, few studies have investigated the interaction between snoRNAs and methylation in EC. In our study, the SNORD11B and SNORD15A were positively correlated with the levels of methylation sites, such as cg16620283 and cg27122942. However, this study only analyzed the correlation between snoRNAs and methylation sites, and the detailed regulatory mechanism between them needs to be further clarified. 
There were some limitations in this study. For example, all results in this study were predicted by the bioinformatics methods, and hence, further experiments and more samples are needed to validate these findings.

\section{Conclusion}

The present study used bioinformatics analyses to screen DE-snoRNAs and DE-SNHGs in EC based on the TCGA dataset. Briefly, DE-snoRNAs of SNORA14B, SNORA47, SNORA71C, SNORD12B, and SNORD14E; DE-SNHGs of SNHG23, SNHG3, SNHG17, SNHG4, and SNHG8, as well as pathways of NODlike receptor signaling pathway, neuroactive ligand-receptor interaction, calcium signaling pathway, cytokine-cytokine receptor interaction, and ECM-receptor interaction, which may be involved the EC development, were obtained. These findings could improve the understanding of the pathogenesis and molecular mechanisms of EC, which will provide effective targets for the treatment of EC.

\section{Abbreviations}

\begin{tabular}{ll} 
Abbreviation & Full term \\
\hline snoRNAs & small nucleolar RNAs \\
\hline SNHGs & small nucleolar RNA host genes \\
\hline TCGA & The Cancer Genome Atlas \\
\hline GO & Gene Ontology \\
\hline KEGG & Kyoto Encyclopedia of Genes and Genomes \\
\hline EC & Esophageal carcinoma \\
\hline ceRNA & competing endogenous RNAs \\
\hline GEO & Gene expression omnibus \\
\hline SD & Standard deviation \\
\hline QL & Quasi-likelihood \\
\hline ESCC & Esophageal squamous cell carcinoma \\
\hline EAC & Esophageal adenocarcinoma
\end{tabular}

\section{Declarations}

Ethics approval and consent to participate

Not Applicable. 
Not applicable.

\section{Competing Interests}

The authors declare that no conflicts of interest exist.

\section{Funding}

This work was supported by the Zhejiang Medical and Health Technology Projects (No.2020RC117).

\section{Authors' Contributions}

All authors participated in the conception and design of the study;

Conceived and drafted the manuscript: Han Shuwen, Jiang Haigen and Zhuang Jing;

Wrote the paper: Han Shuwen, Yang Xi and Zhuang Jing;

Analysed the data: Wu Wei, Dai Siqi and Jiang Haigen;

Drew figures: Zhuang Jing and Qi Quan.

All authors read and approved the paper.

\section{Acknowledgements}

The authors gratefully acknowledge the database available to us for this study.

\section{Availability of data and materials}

The datasets generated during the current study are not publicly available but obtained from corresponding authors on reasonable request.

\section{References}

1. Fitzmaurice C, Dicker D, Pain A, Hamavid H, Moradi-Lakeh M, et al. (2015) The global burden of cancer 2013. JAMA oncology 1: 505-527.

2. Bray F, Ferlay J, Soerjomataram I, Siegel RL, Torre LA, et al. (2018) Global cancer statistics 2018: GLOBOCAN estimates of incidence and mortality worldwide for 36 cancers in 185 countries. CA: a cancer journal for clinicians 68: 394-424.

3. Siegal R, Naishadham D, Jemal A (2012) Cancer statistics, 2012. Ca Cancer J Clin 62: 10-29.

4. Coleman HG, Xie S-H, Lagergren J (2018) The epidemiology of esophageal adenocarcinoma. Gastroenterology 154: 390-405. 
5. Abnet CC, Arnold M, Wei W-Q (2018) Epidemiology of esophageal squamous cell carcinoma. Gastroenterology 154: 360-373.

6. Rustgi AK, El-Serag HB (2014) Esophageal carcinoma. New England Journal of Medicine 371: 24992509.

7. Chang L-S, Lin S-Y, Lieu A-S, Wu T-L (2002) Differential expression of human 5 S snoRNA genes. Biochemical and biophysical research communications 299: 196-200.

8. McMahon M, Contreras A, Ruggero D (2015) Small RNAs with big implications: new insights into H/ACA snoRNA function and their role in human disease. Wiley Interdisciplinary Reviews: RNA 6: 173-189.

9. Serrano-Gomez SJ, Sanabria-Salas MC, Garay J, Baddoo MC, Hernandez-Suarez G, et al. (2017) Ancestry as a potential modifier of gene expression in breast tumors from Colombian women. PloS one 12: e0183179.

10. Gong J, Li Y, Liu C-j, Xiang Y, Li C, et al. (2017) A pan-cancer analysis of the expression and clinical relevance of small nucleolar RNAs in human cancer. Cell reports 21: 1968-1981.

11. López-Corral L, Mateos MV, Corchete LA, Sarasquete ME, de la Rubia J, et al. (2012) Genomic analysis of high-risk smoldering multiple myeloma. Haematologica 97: 1439-1443.

12. Patterson DG, Roberts JT, King VM, Houserova D, Barnhill EC, et al. (2017) Human snoRNA-93 is processed into a microRNA-like RNA that promotes breast cancer cell invasion. NPJ Breast Cancer 3 : 25.

13. Gong J, Li Y, Liu C-j, Xiang Y, Li C, et al. A Pan-cancer Analysis of the Expression and Clinical Relevance of Small Nucleolar RNAs in Human Cancer. Cell Reports 21: 1968-1981.

14. Williams GT, Farzaneh F (2012) Are snoRNAs and snoRNA host genes new players in cancer? Nature reviews cancer 12: 84 .

15. Song H, Song J, Lu L, Li S (2019) SNHG8 is upregulated in esophageal squamous cell carcinoma and directly sponges microRNA-411 to increase oncogenicity by upregulating KPNA2. OncoTargets and therapy 12: 6991.

16. Zhang $Y$, Jin X, Wang Z, Zhang X, Liu S, et al. (2018) Downregulation of SNHG1 suppresses cell proliferation and invasion by regulating Notch signaling pathway in esophageal squamous cell cancer. Cancer Biomarkers 21: 89-96.

17. Goldman M, Craft B, Brooks A, Zhu J, Haussler D (2018) The UCSC Xena Platform for cancer genomics data visualization and interpretation. BioRxiv: 326470.

18. Robinson MD, McCarthy DJ, Smyth GK (2010) edgeR: a Bioconductor package for differential expression analysis of digital gene expression data. Bioinformatics 26: 139-140.

19. Ritchie ME, Phipson B, Wu D, Hu Y, Law CW, et al. (2015) limma powers differential expression analyses for RNA-sequencing and microarray studies. Nucleic acids research 43: e47-e47.

20. Ernst J, Bar-Joseph Z (2006) STEM: a tool for the analysis of short time series gene expression data. BMC bioinformatics 7: 191. 
21. Yoshihama M, Nakao A, Kenmochi N (2013) snOPY: a small nucleolar RNA orthological gene database. Bmc Research Notes 6: 1-5.

22. Yu G, Wang L-G, Han Y, He Q-Y (2012) clusterProfiler: an R package for comparing biological themes among gene clusters. Omics: a journal of integrative biology 16: 284-287.

23. Thorenoor N, Slaby O (2015) Small nucleolar RNAs functioning and potential roles in cancer. Tumor Biology 36: 41-53.

24. Li C, Zhou L, He J, Fang X-Q, Zhu S-W, et al. (2016) Increased long noncoding RNA SNHG20 predicts poor prognosis in colorectal cancer. BMC cancer 16: 655.

25. Ronchetti D, Mosca L, Cutrona G, Tuana G, Gentile M, et al. (2013) Small nucleolar RNAs as new biomarkers in chronic lymphocytic leukemia. BMC medical genomics 6: 27.

26. Braicu C, Zimta A-A, Harangus A, lurca I, Irimie A, et al. (2019) The function of non-coding RNAs in lung cancer tumorigenesis. Cancers 11: 605.

27. Li G, He Y, Liu X, Zheng Z, Zhang M, et al. (2017) Small nucleolar RNA 47 promotes tumorigenesis by regulating EMT markers in hepatocellular carcinoma. Minerva medica 108: 396-404.

28. Schulten H-J, Bangash M, Karim S, Dallol A, Hussein D, et al. (2017) Comprehensive molecular biomarker identification in breast cancer brain metastases. Journal of translational medicine 15: 269.

29. Liu Z, Tao H (2019) Small nucleolar RNA host gene 3 facilitates cell proliferation and migration in oral squamous cell carcinoma via targeting nuclear transcription factor $Y$ subunit gamma. Journal of cellular biochemistry.

30. Liu J, Li W, Zhang J, Ma Z, Wu X, et al. (2019) Identification of key genes and long non-coding RNA associated ceRNA networks in hepatocellular carcinoma. PeerJ 7: e8021.

31. Gao H, Liu R, Sun X (2019) STAT3-induced upregulation of IncRNA SNHG17 predicts a poor prognosis of melanoma and promotes cell proliferation and metastasis through regulating PI3K-AKT pathway. European review for medical and pharmacological sciences 23: 8000-8010.

32. Xu T, Yan S, Jiang L, Yu S, Lei T, et al. (2019) Gene Amplification-Driven Long Noncoding RNA SNHG17 Regulates Cell Proliferation and Migration in Human Non-Small-Cell Lung Cancer. Molecular Therapy-Nucleic Acids 17: 405-413.

33. Zhang G, Xu Y, Wang S, Gong Z, Zou C, et al. (2019) LncRNA SNHG17 promotes gastric cancer progression by epigenetically silencing of $p 15$ and p57. Journal of cellular physiology 234: 51635174.

34. Liu D (2015) LYN, a key gene from bioinformatics analysis, contributes to development and progression of esophageal adenocarcinoma. Medical science monitor basic research 21: 253.

35. Zeng J-H, Xiong D-D, Pang Y-Y, Zhang Y, Tang R-X, et al. (2017) Identification of molecular targets for esophageal carcinoma diagnosis using miRNA-seq and RNA-seq data from The Cancer Genome Atlas: A study of 187 cases. Oncotarget 8: 35681. 
36. Zhu W, Niu J, He M, Zhang L, Lv X, et al. (2019) SNORD89 promotes stemness phenotype of ovarian cancer cells by regulating Notch1-c-Myc pathway. Journal of translational medicine 17: 259.

37. Sun Y, Chen E, Li Y, Ye D, Cai Y, et al. (2019) H/ACA box small nucleolar RNA 7B acts as an oncogene and a potential prognostic biomarker in breast cancer. Cancer cell international 19: 125.

38. Kalikawe R, Baba Y, Nomoto D, Okadome K, Miyake K, et al. (2019) Lysyl oxidase impacts disease outcomes and correlates with global DNA hypomethylation in esophageal cancer. Cancer science 110: 3727.

39. Iwabu J, Yamashita S, Takeshima H, Kishino T, Takahashi T, et al. (2019) FGF5 methylation is a sensitivity marker of esophageal squamous cell carcinoma to definitive chemoradiotherapy. Scientific reports 9: 1-10.

\section{Tables}

Table 1 The clinicopathological parameters of the patients

\begin{tabular}{llllll}
\hline & I & II & III & IV & P value \\
\hline & $67.95 \pm 11.93$ & $61.79 \pm 12.37$ & $62.78 \pm 11.69$ & $59.63 \pm 7.89$ & 0.27 \\
tale & 11 & 59 & 48 & 8 & $<0.001$ \\
smale & 10 & 12 & 3 & 0 & \\
sophagus & 21 & 70 & 50 & 8 & 0.91 \\
tomach & 0 & 1 & 1 & 0 & \\
denomas and Adenocarcinomas & 13 & 24 & 29 & 5 & 0.02 \\
quamous Cell Neoplasms & 8 & 47 & 22 & 3 & \\
& $29.63 \pm 6.78$ & $24.59 \pm 5.92$ & $24.10 \pm 4.35$ & $24.68 \pm 5.70$ & 0.16 \\
\hline
\end{tabular}

BMI: body mass index; I, II, III and IV means the clinical stages of esophageal carcinoma; $\mathrm{P}<0.05$ was considered to be statistically significant difference.

Table 2 The host genes of the DE-snoRNAs and snoRNAs related to clinical stage of EC 


\begin{tabular}{|c|c|c|c|c|}
\hline snoRNA name & Species & Target RNA & Locus & name2 \\
\hline \multicolumn{5}{|l|}{ DE-snoRNAs } \\
\hline SNORA14A & Homo_sapiens & 18S rRNA & POR & ACA14a \\
\hline SNORD11B & Homo_sapiens & 18S rRNA & NOP5/NOP58 & HBII-95B \\
\hline SNORD12B & Homo_sapiens & 28S rRNA & C20orf199 & HBII-99B \\
\hline SNORD15A & Homo_sapiens & 28S rRNA & RPS3 & U15A \\
\hline SNORD53 & Homo_sapiens & 28S rRNA & WDR43 & U53 \\
\hline SNORD72 & Homo_sapiens & 28S rRNA & RPL37 & HBII-240 \\
\hline SNORD88A & Homo_sapiens & 28S rRNA & C19orf48 & HBII-180A \\
\hline SNORD99 & Homo_sapiens & 28S rRNA & C1orf79 & HBII-420 \\
\hline SCARNA10 & Homo_sapiens & U5 snRNA & NCAPD2 & U85 \\
\hline SCARNA12 & Homo_sapiens & U5 snRNA & PHB2 & U89 \\
\hline SNORA1 & Homo_sapiens & 28S rRNA & JOSD3 & ACA1 \\
\hline SNORA11 & Homo_sapiens & Unknown & MAGED2 & U107 \\
\hline SNORA12 & Homo_sapiens & Unknown & CWF19L1 & U108 \\
\hline SNORA13 & Homo_sapiens & 18S rRNA & C5orf26 & ACA13 \\
\hline
\end{tabular}

Box column, indicating the type of snoRNA

Traget RNA column, indicating snoRNA's target RNA

Organization column, representing snoRNA comes from introns

Locus, indicating the source gene of snoRNA.

\section{Supplemental Information Note}

Supplementary Figure 1 The main workflow of this study.

\section{Figures}



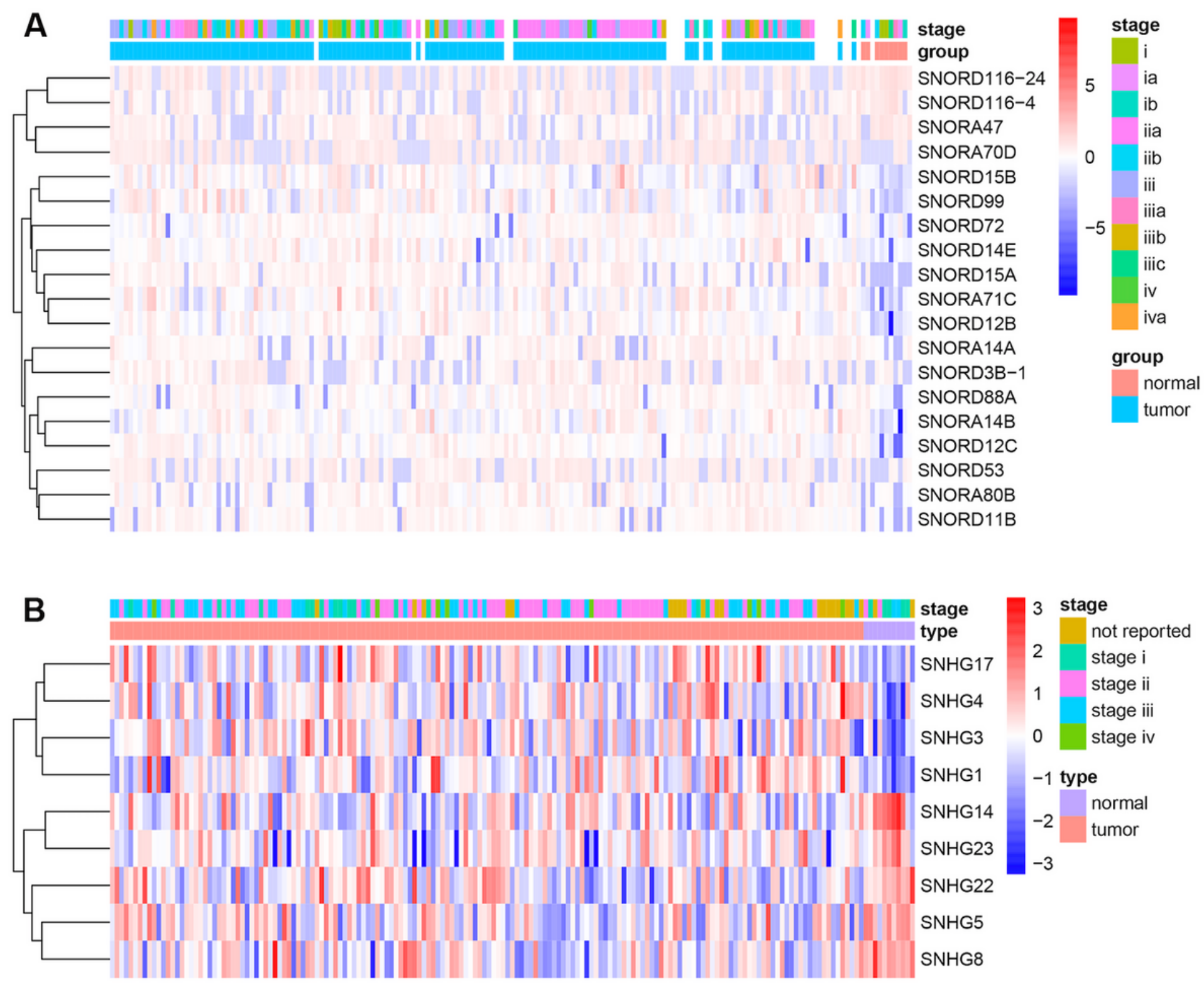

Figure 1

Screening of the differentially expressed snoRNA and SNHGs between esophageal carcinoma and normal controls. The heat map of hierarchical clustering analysis indicating the differential snoRNA (A) or SNHGs (B). 3, 2, 1, 0, -1, -2, and -3 are fold changes compared with normal samples. 
A
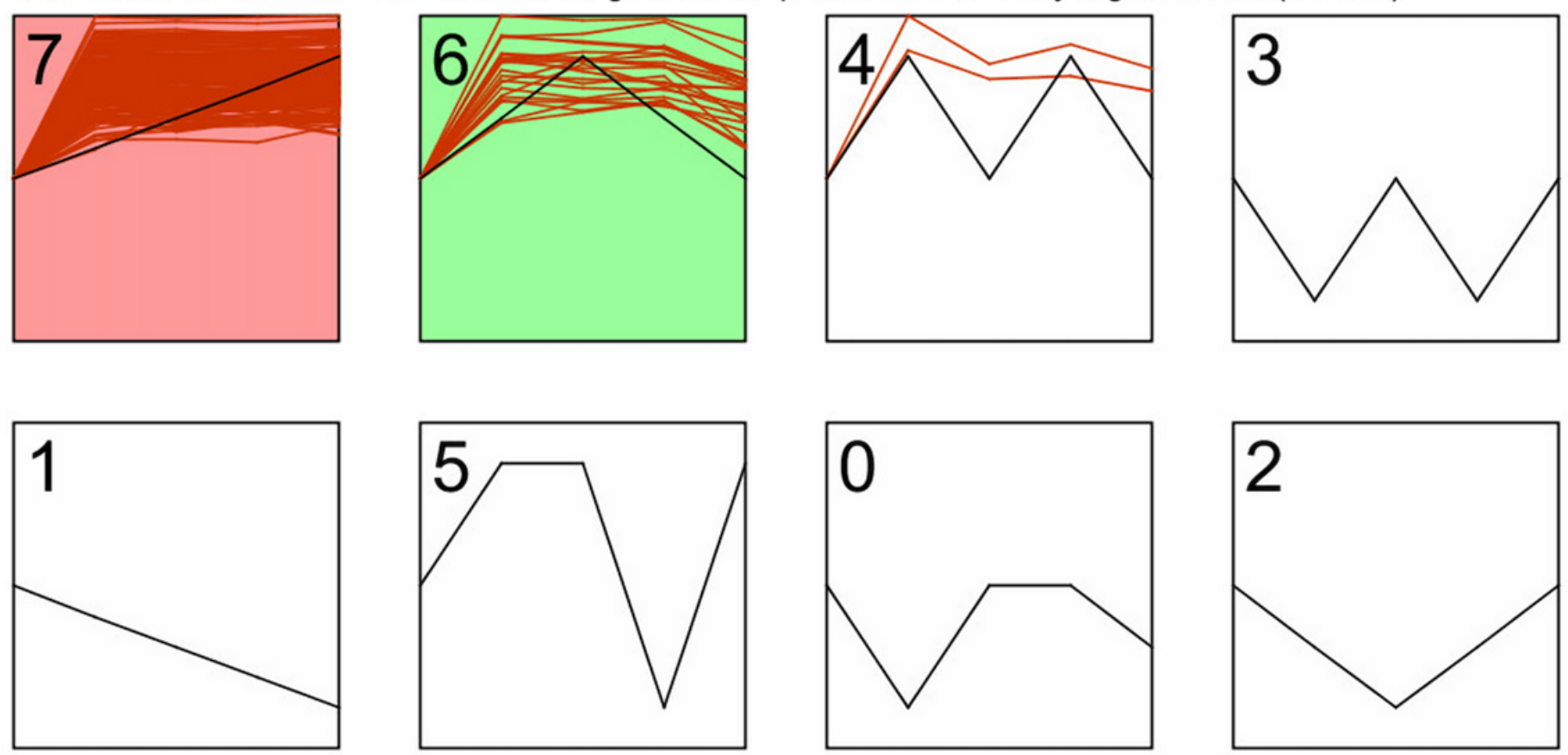

B

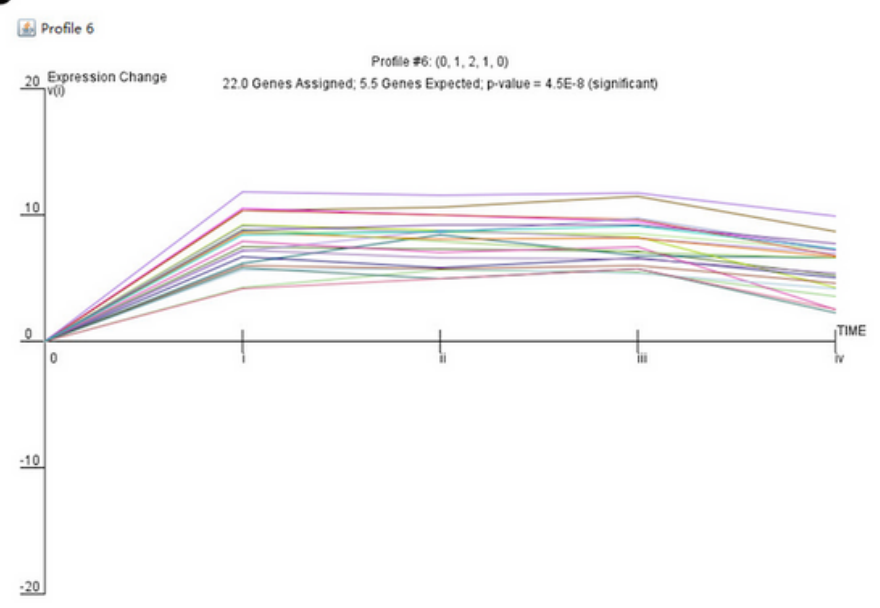

C

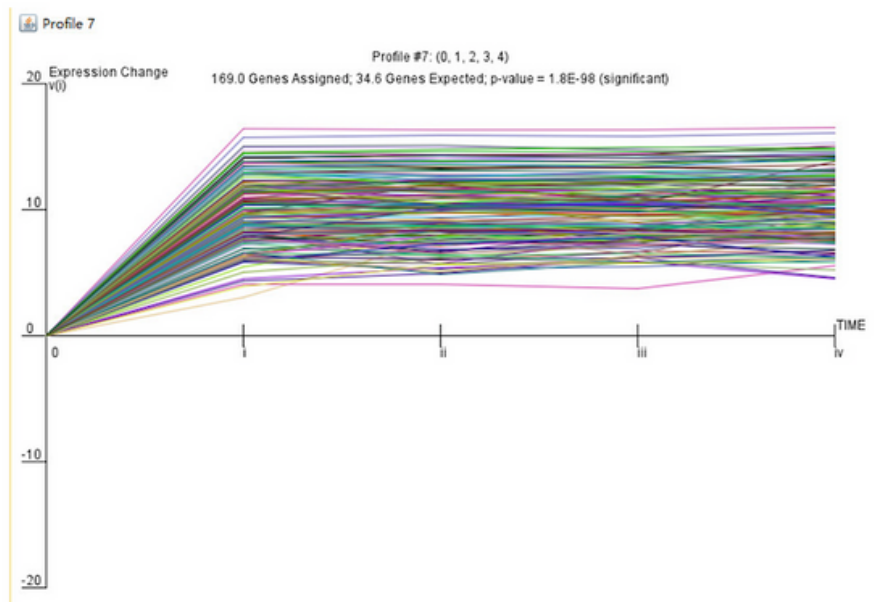

Figure 2

The expression trends of snoRNAs related to clinical stage of esophageal carcinoma. (A) Trend analysis revealed that cluster 7 and cluster 6 are the significant modules. The colored line represents the change of the expression trend of a gene at different time points. The black line represents the general trend of changes in these genes. $(B, C)$ The expression of each gene is shown according to the stage node in cluster 7 and cluster 6 , respectively. Profile 7 and 6 represent cluster 7 and cluster 6 , respectively. 
A
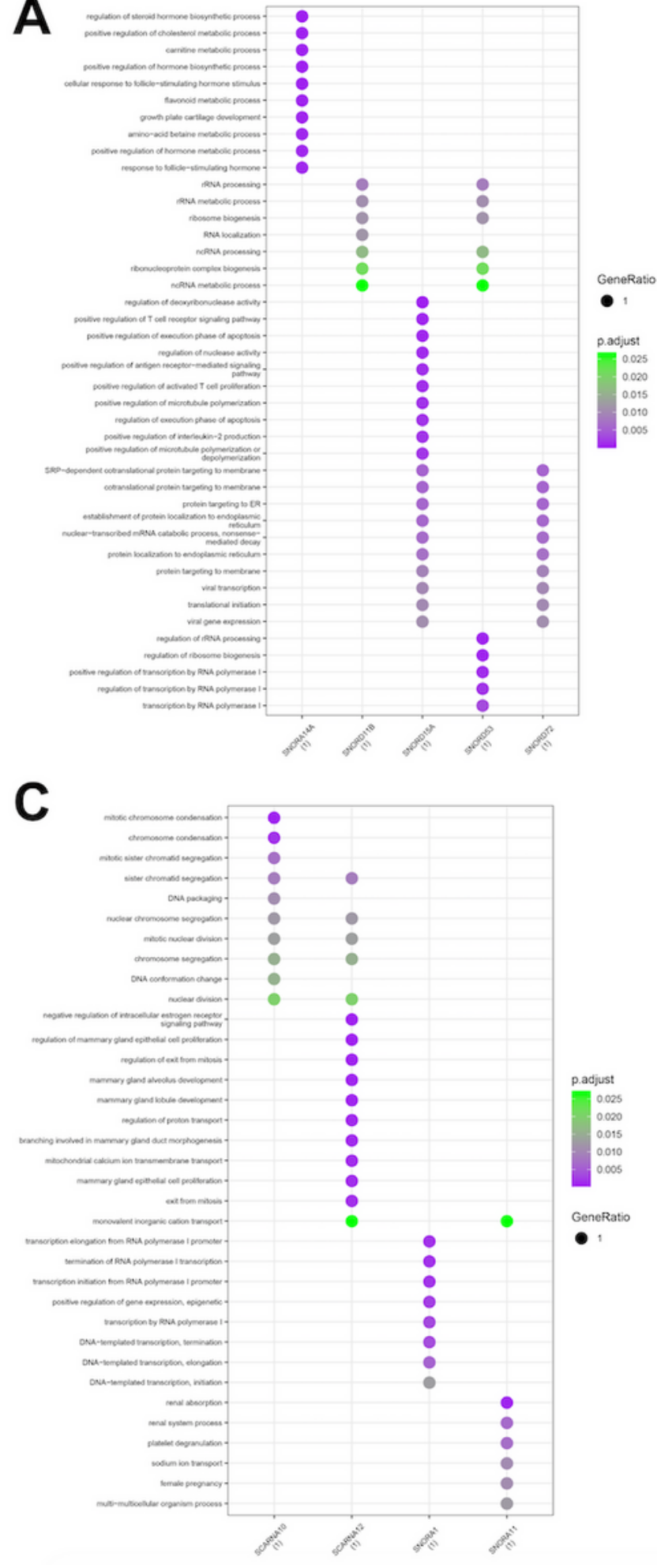

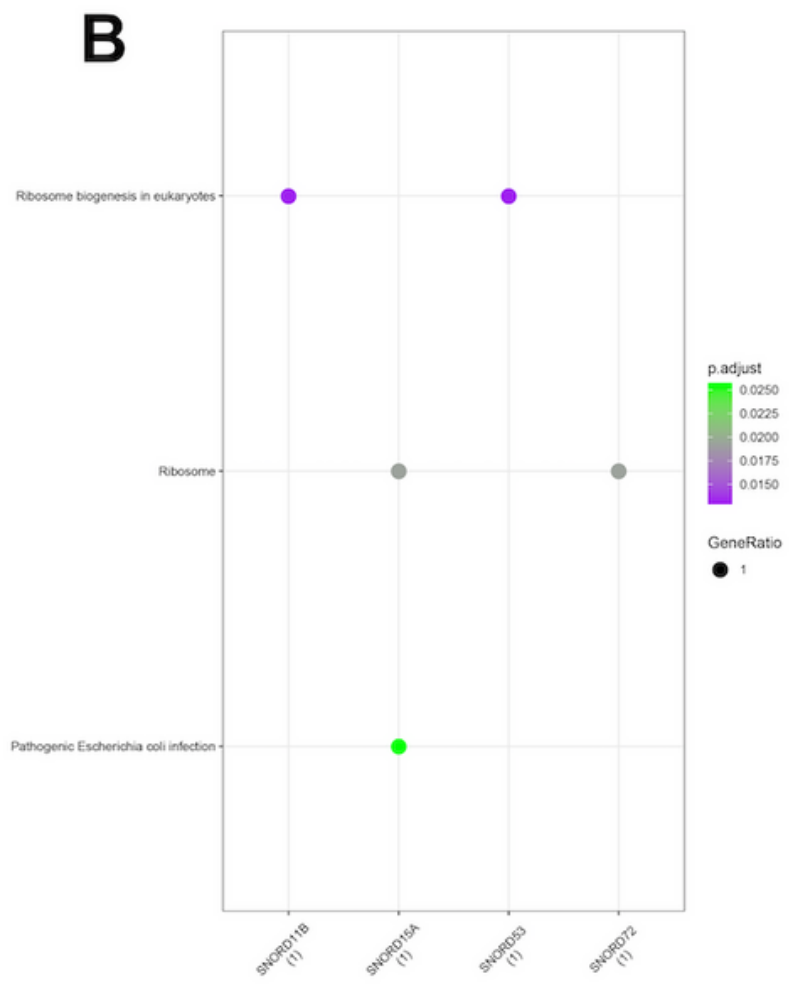

\section{Figure 3}

Enrichment analysis for the host genes of DE-snoRNAs and snoRNAs related to clinical stage of EC. (A) $\mathrm{GO}$ analysis revealed significantly enriched biological processes of the host genes of DE-snoRNAs. (B, C) KEGG pathway analysis revealed significantly enriched pathways for host genes of DE-snoRNAs and snoRNAs related to clinical stage of EC, respectively. GO, Gene Ontology; KEGG, Kyoto Encyclopedia of Genes and Genomes. 

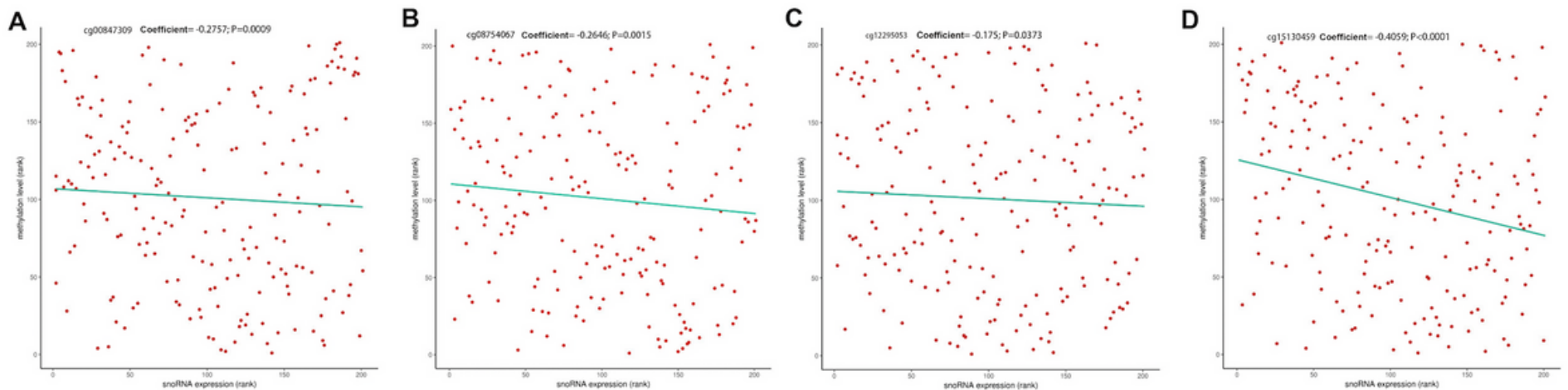

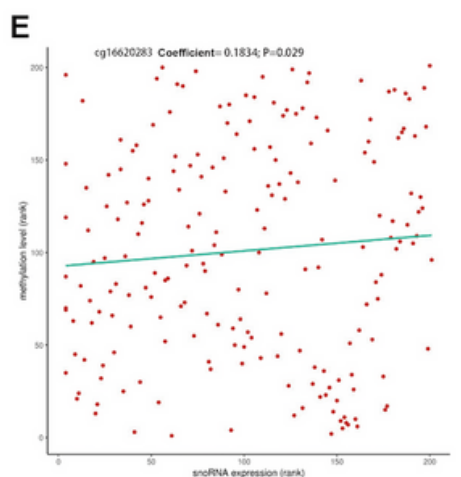

$\mathbf{F}$
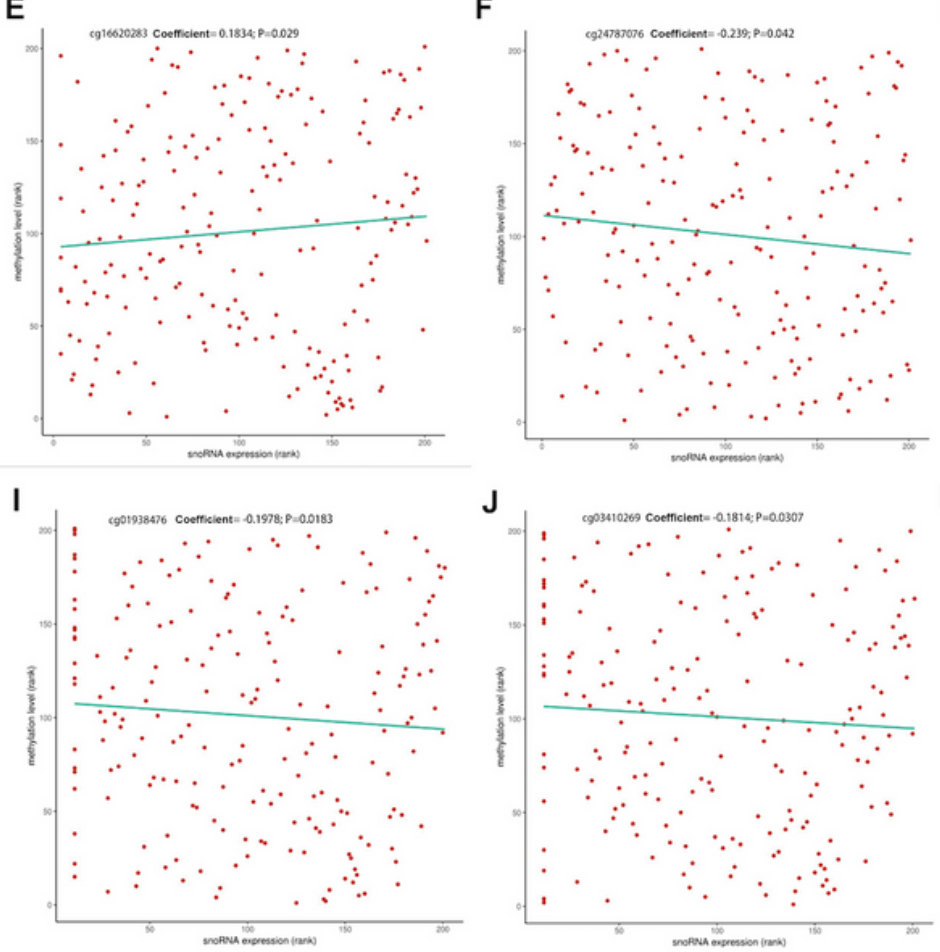

J

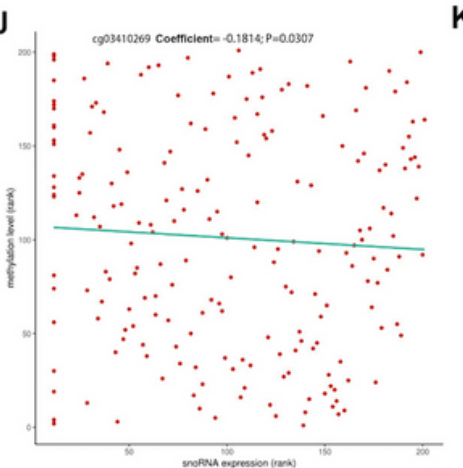

G

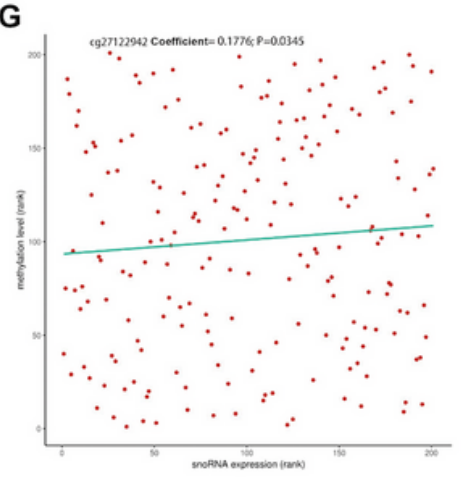

K

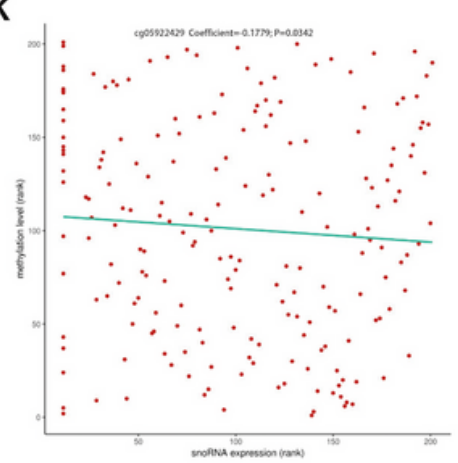

H

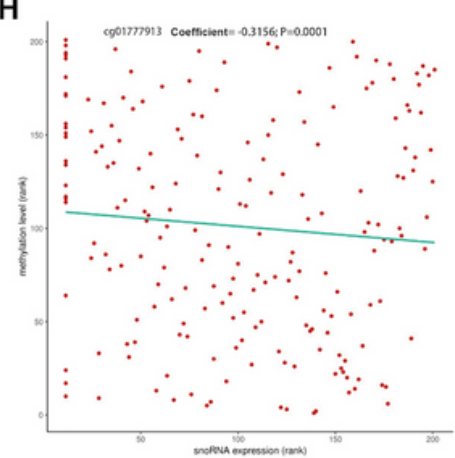

L

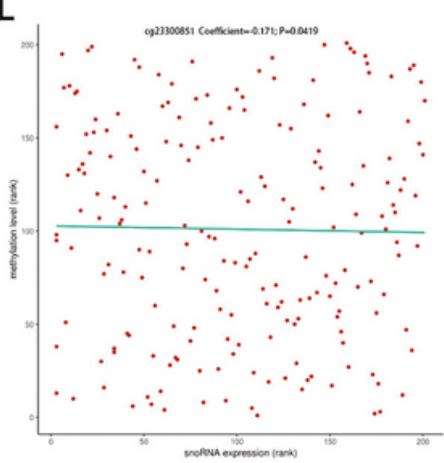

Figure 4

Correlation analysis between snoRNAs and methylation. A total of 7 DE-sonRNAs (A-G) and 5 snoRNAs related to clinical stage $(\mathrm{H}-\mathrm{L})$ were significantly correlated with the methylation levels. 


\section{Strata + Expression=High $\leftarrow$ Expression=Low}

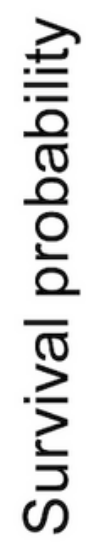

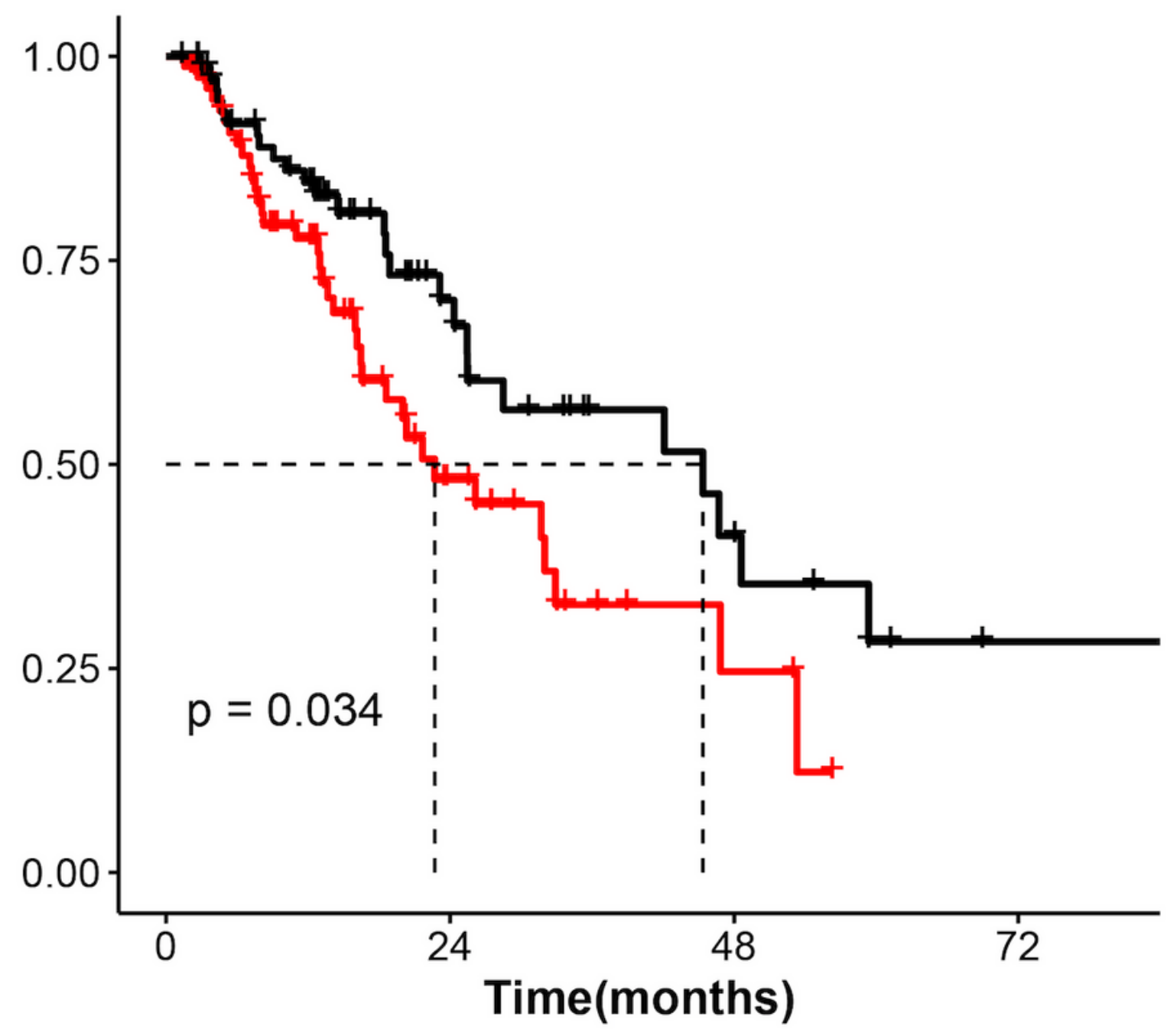

Number at risk

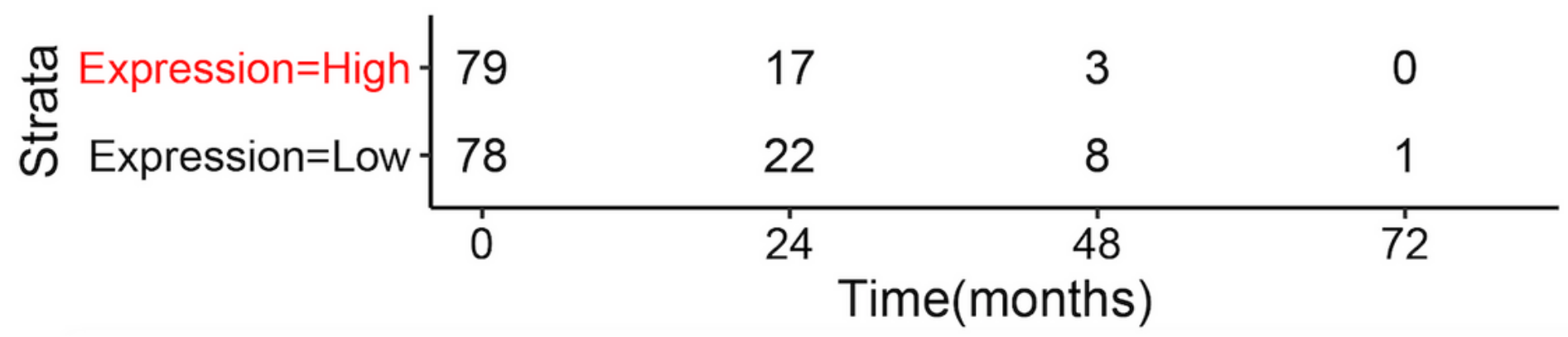

Figure 5

Survival analysis of SNORA70D. Kaplan-Meier curve showed that patients with high expression of SNORA70D had shorter overall survival compared to those with low expression. 

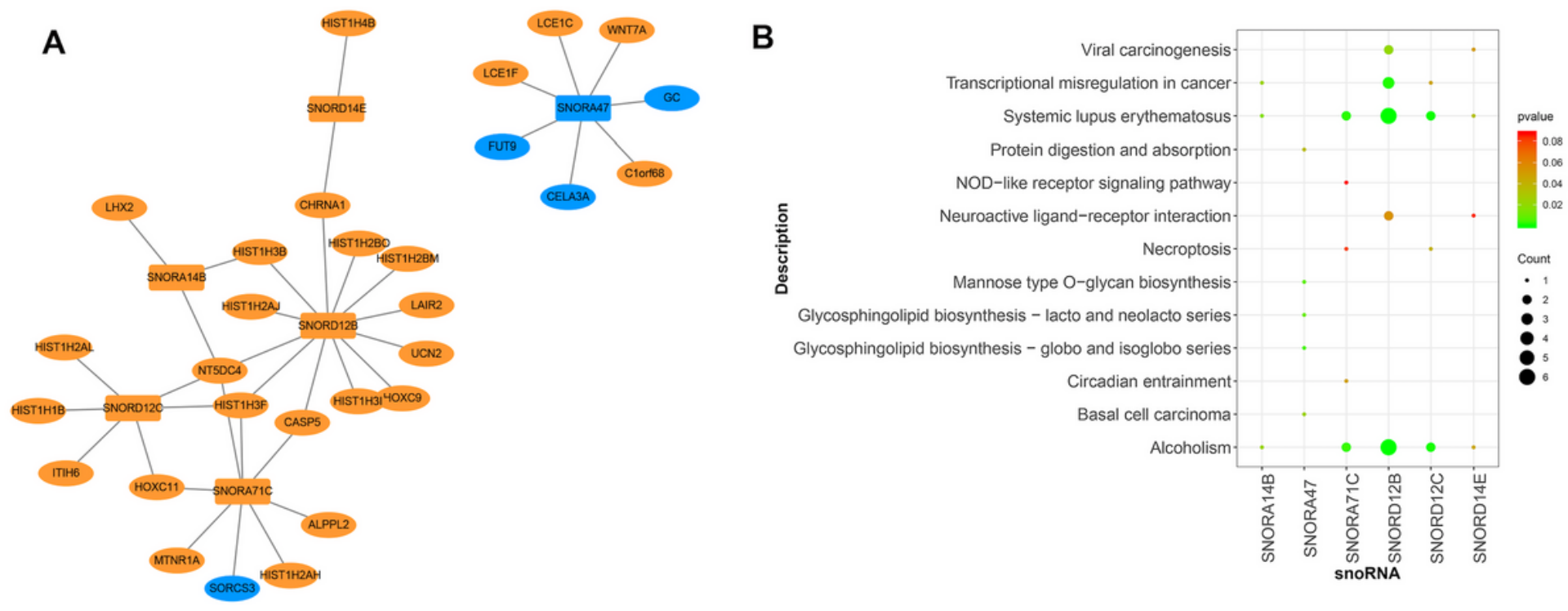

Figure 6

The snoRNA -mRNA co-expression network and KEGG enrichment analysis. A: Yellow and blue of oval represent up- and down-regulated mRNA. The rectangle represents snoRNA. B: The horizontal axis represents snoRNAs, and the vertical axis represents enriched pathways.
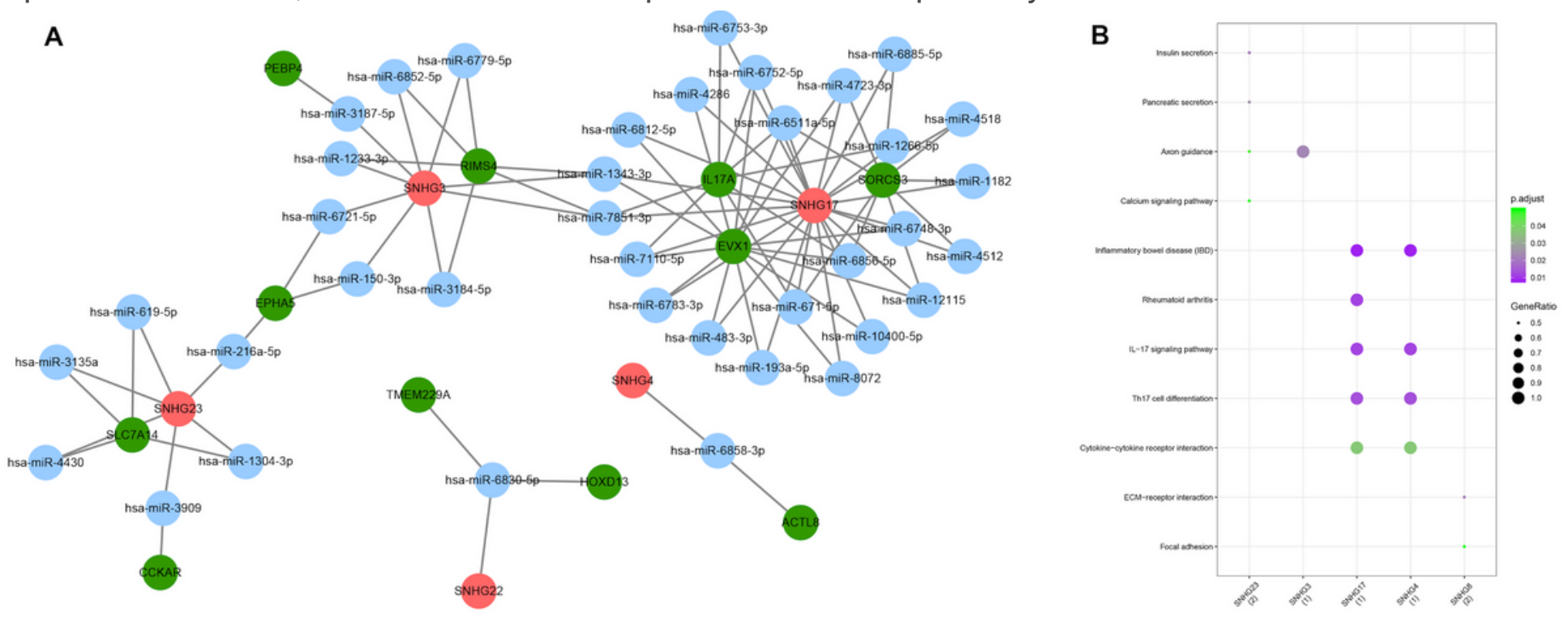

Figure 7

The ceRNA network and KEGG enrichment analysis. A: The red, green and blue circle represents SNHGs, mRNA, and miRNA, respectively. B: The horizontal axis represents SNHGs, and the vertical axis represents enriched pathways. The ceRNA network and KEGG enrichment analysis. A: The red, green and blue circle represents SNHGs, mRNA, and miRNA, respectively. B: The horizontal axis represents SNHGs, and the vertical axis represents enriched pathways.

\section{Supplementary Files}


This is a list of supplementary files associated with this preprint. Click to download.

- Supplementarytables.docx

- ESCAlncRNAtop5.fa.out.result.txt

- ESCAstageprofile6.txt

- ESCAstageprofile7.txt

- Supplementaryfigure1.tif

- ESCAmRNAtop5.fa.out.result.txt 